\title{
Dietary Anthocyanins against Obesity and Inflammation
}

\author{
Yoon-Mi Lee ${ }^{1,2}$, Young Yoon ${ }^{1}$, Haelim Yoon ${ }^{1}$, Hyun-Min Park ${ }^{1}$, Sooji Song ${ }^{1}$ \\ and Kyung-Jin Yeum 1,3,* \\ 1 Division of Food Bioscience, College of Biomedical and Health Sciences, Konkuk University, Chungju-si \\ 27478, Korea; yoonmilee@kku.ac.kr (Y.-M.L.); lab_yyoung0418@naver.com (Y.Y.); limtiny@naver.com (H.Y.); \\ loveangela0312@gmail.com (H.-M.P.); ssj4037@naver.com (S.S.) \\ 2 Nanotechnology Research Center, Konkuk University, Chungju-si 27478, Korea \\ 3 Institute of Biomedical and Health Science, Konkuk University, Chungju-si 27478, Korea \\ * Correspondence: kyeum@kku.ac.kr; Tel.: +82-43-840-3586; Fax: +82-43-840-3585
}

Received: 14 August 2017; Accepted: 25 September 2017; Published: 1 October 2017

\begin{abstract}
Chronic low-grade inflammation plays a pivotal role in the pathogenesis of obesity, due to its associated chronic diseases such as type II diabetes, cardiovascular diseases, pulmonary diseases and cancer. Thus, targeting inflammation is an attractive strategy to counter the burden of obesity-induced health problems. Recently, food-derived bioactive compounds have been spotlighted as a regulator against various chronic diseases due to their low toxicity, as opposed to drugs that induce severe side effects. Here we describe the beneficial effects of dietary anthocyanins on obesity-induced metabolic disorders and inflammation. Red cabbage microgreen, blueberry, blackcurrant, mulberry, cherry, black elderberry, black soybean, chokeberry and jaboticaba peel contain a variety of anthocyanins including cyanidins, delphinidins, malvidins, pelargonidins, peonidins and petunidins, and have been reported to alter both metabolic markers and inflammatory markers in cells, animals, and humans. This review discusses the interplay between inflammation and obesity, and their subsequent regulation via the use of dietary anthocyanins, suggesting an alternative dietary strategy to ameliorate obesity and obesity associated chronic diseases.
\end{abstract}

Keywords: obesity; inflammation; anthocyanin; flavonoids

\section{Introduction}

Obesity is accompanied by metabolic disturbances such as dyslipidemia, hyperglycemia and hypertension. The burden of obesity is largely derived from its associated chronic diseases such as cardiovascular diseases, pulmonary diseases, cancer, and type II diabetes mellitus [1,2]. Continuous effort has been made to understand the pathogenesis between obesity and chronic diseases. Interestingly, chronic low-grade inflammation has been linked to the progression of obesity and its related diseases [3,4].

Inflammation is a defense system that responds to harmful stimuli and restores disruptive tissues back to homeostasis [5]. Upon recognizing unfavorable stimuli, including excessive nutrients, immune cells urgently secrete a number of cytokines and chemokines to the damaged area, defined as innate immunity. If the condition is prolonged, antigen-presenting cells (APC) and B- and $\mathrm{T}$ lymphocytes perform adaptive immunity which may increase the prevalence of obesity-associated chronic diseases [6]. Thus, preventing this prolonged pro-inflammatory condition can be a valid strategy against obesity-associated metabolic disorders [7,8].

Although there are a number of drugs that are approved for the treatment of obese patients, many of them were withdrawn due to the severe adverse events such as heart diseases and psychiatric disorders [9]. Meanwhile, recent studies demonstrated that consumption of food-derived bioactive 
components such as phenolic compounds is positively associated with reducing the risk of obesity and its associated chronic diseases with low toxicity $[10,11]$. Thus, creating new dietary treatments based on various bioactive components in food has been emerging as a new possible intervention against obesity [12,13].

Anthocyanins are an important subfamily of flavonoids, which are abundant in flowers, fruits, seeds and plant leaves [14]. The basic structural form 2-phenylchromenylium is further classified into six major compounds: cyanidin, delphinidin, malvidin, pelargonidin, peonidin and petunidin, depending on the flavylium B-ring. Sugars (glucose, arabinose, galactose, etc.) can be attached to the main structure of anthocyanins [12,15] (Figure 1). Because anthocyanins are commonly consumed, their biological activities have been extensively studied. Notably, anthocyanins have antimicrobial, antioxidative, anti-inflammatory, and anti-mutagenic properties, which in turn play a role on the prevention and treatment of many chronic diseases such as metabolic disorders, cancer, eye diseases and cardiovascular diseases [12,16,17]. In particular, mixtures of anthocyanins found in food rather than their individual anthocyanin components has been reported to be more beneficial for improving human health [18].

\begin{tabular}{|l|l|l|l|l|} 
sugar \\
\hline
\end{tabular}

Figure 1. Structure of the most common anthocyanins.

Collectively, over the last two decades, the link between obesity and inflammation has been highlighted as a new axis to be targeted for intervention. Dietary bioactive components that have beneficial effects against both obesity and inflammation can potentially be a modulator of this axis. Here we discuss the impact of dietary anthocyanins on alleviating obesity and inflammation.

\section{Metainflammation and Its Mechanism}

Obesity is accompanied by a chronic low-grade inflammatory condition known as metainflammation $[19,20]$. Pro-inflammatory markers such as interleukin-6 (IL-6), interleukin-1 $\beta$ (IL-1 $\beta)$, C-reactive protein (CRP) and tumor necrosis factor- $\alpha$ (TNF- $\alpha$ ) were reported to be persistently expressed in adipocytes of white adipose tissue [19]. It is interesting to note that pro-inflammatory markers were significantly higher in obese patients compared to healthy individuals. Furthermore, obese patients with high IL-6 levels also had increased CRP levels, which is a critical risk factor for cardiovascular diseases and type 2 diabetes [13,21]. In addition, M1 macrophages, T cells, B cells and monocyte chemoattractant protein-1 (MCP-1) were also increased in adipose tissue, whereas there was a reduction in the number of regulatory T cells, M2 macrophages and amount of adiponectin [22]. The possible underlying mechanism is that toll-like receptors (TLRs), in particular TLR4, recognize external stimuli including over-nutrition, and connect them to transcription factors such as nuclear factor Kappa B (NF-кB), activated protein-1 (AP-1) and interferon regulatory factor 3 (IRF3). These inflammation-associated transcription factors enter into the nucleus and bind to the site of target genes that promote inflammation [23]. Due to the coordination between the immune system and metabolism, developing novel immunotherapeutic strategies to simultaneously attenuate obesity-related metabolic disorders seems promising. Indeed, targeting IL-1 $\beta$, IL- $1 \beta$ receptor, TNF- $\alpha$, TLRs, T and B lymphocytes were effective in reducing blood glucose levels, insulin resistance and other metabolic markers in 
obesity $[7,8]$. Flavonoids have also been assessed to regulate inflammation and obesity by lowering IL- $1 \alpha$, and IFN- $\gamma$ compared to control mice [24]. These efforts have diversely been attempted using cell, animal and human models [25].

\section{Establishment of an Obesity Model}

Cells and animals are essential surrogates for human obesity due to the limitations of promising human studies. In this section, we introduce frequently used cell and animal models of human obesity (Table 1).

Table 1. Frequently used obesity model in vitro and in vivo.

\begin{tabular}{|c|c|c|c|c|}
\hline & & & Advantage & Disadvantage \\
\hline \multirow[b]{2}{*}{ In vitro } & mouse & $\begin{array}{l}\text { mesenchymal stem cells } \\
\text { embryonic stem cells } \\
\text { primary preadipocytes }\end{array}$ & $\begin{array}{l}\text { - } \quad \text { easy to } \\
\text { study mechanism, } \\
\text { - } \quad \text { well-established method }\end{array}$ & $\begin{array}{l}\text { different characteristics } \\
\text { of human vs. mouse }\end{array}$ \\
\hline & human & $\begin{array}{l}\text { preadipocytes } \\
\text { adipose-derived stem } \\
\text { cells }\end{array}$ & $\begin{array}{l}\text { - } \quad \text { easy to } \\
\text { study mechanism, } \\
\text { representation of } \\
\text { human obesity }\end{array}$ & $\begin{array}{l}\text { - complement of mouse } \\
\text { cell lines' disadvantages }\end{array}$ \\
\hline \multirow{2}{*}{ In vivo } & monogenic & $\begin{array}{l}\text { ob/ob mice } \\
\text { Zucker fatty rats }\end{array}$ & $\begin{array}{l}\text { apparent symptoms of } \\
\text { metabolic disorders }\end{array}$ & $\begin{array}{l}\text { - monogenic mutation is } \\
\text { rare in humans }\end{array}$ \\
\hline & polygenic & $\begin{array}{l}\text { High energy diets-fed } \\
\text { mice } \\
\text { Diet-induced obesity }\end{array}$ & $\begin{array}{l}\text { - } \quad \text { resemble complexities of } \\
\text { human obesity }\end{array}$ & $\begin{array}{l}\text { - complement of } \\
\text { monogenic } \\
\text { models' disadvantages }\end{array}$ \\
\hline
\end{tabular}

Fat accumulation in adipose tissue is critical for obesity. Adipose tissue is composed of adipocytes, macrophages, mesenchymal stem cells, blood cells, fibroblasts, pericytes, and smooth muscle cells [26]. Cells are important in order to identify the mechanism of key adipogenic process. Mouse cell lines to study adipogenesis can be classified into three categories: mesenchymal stem cells, embryonic cells, and primary preadipocytes [27]. Among them, 3T3-L1 is the most frequently used cell line, due to its well-established methodology for mature adipocytes. When adipogenic stimuli such as insulin (1 to $10 \mu \mathrm{g} / \mathrm{mL})$, dexamethasone, $(1 \mu \mathrm{M})$ and 3-isobutyl-1-methylxanthine (IBMX) $(0.5 \mathrm{mM})$ are added to medium containing $10 \%$ fetal bovine serum, several transcriptional factors such as peroxisome proliferator-activated receptor gamma (PPAR- $\gamma$ ), CCAAT/enhancer-binding proteins (C/EBPs) and sterol regulatory element binding (SREBP) promote adipocyte fate, resulting in fat accumulation in mature adipocyte within 14 days [28]. Mouse cell models can be easily applied to study adipogenesis in obesity, but there are some metabolic differences with human adipocytes [29]. Therefore, human preadipocyte and adipose-derived stem cells were developed, which also require adipogenic stimuli (Insulin, dexamethasone, IBMX, biotin, rosiglitazone) for differentiation to mature adipocytes by transcriptional cascades within 12-14 days [30]. Recent studies of obesity in vitro have utilized not only the above described cell models but also utilized co-culture and three dimensional culture systems [31].

Obese animals can be categorized into two groups, monogenic and polygenic models [32]. In monogenic models, ob/ob mice are C57BL/ 6 that spontaneously become obese due to a mutation in the leptin gene responsible for controlling appetite [33,34]. Zucker rats are a monogenic obese model where the rats are autosomal recessive for a mutation in leptin receptors, and consequently exhibit metabolic symptoms such as hyperglycemia and insulin resistance [35]. Despite the metabolic disorder phenotype displayed in these monogenic models, these models are limited in their representation of human obesity since they are induced via a mutation of a gene [36]. Diet-induced obesity models have been alternatively suggested to overcome this limitation. These animals are instead fed with high energy diets such as high fat ( $45 \%$ or $60 \%$ kcal energy from fat as per difference of lard contents), 
high-fructose and high-cholesterol diets. C57BL/6 and A/J mice are commonly used [37]. Wistar, Sprague-Dawley, Long Evans and Osborne mendel rat species are commonly used as high energy diet-induced obese rat model [38]. These polygenic models developed by high energy diets have shown the characteristic symptoms of metabolic disorders such as glucose intolerance and upregulation of cholesterol and triglycerides in the plasma of animals [32]. Diet-induced obese rats are another polygenic model where rats have been bred selectively through several generations to become obese without high energy diet supplementation [39]. These valid models may lead us to predict the mechanism and efficacy of target molecules for human obesity.

\section{Biological Functions of Anthocyanins}

Anthocyanins are well-known antioxidants that eliminate reactive oxygen species (ROS). It has been proven that anthocyanins, in particular cyanidin-3-glucoside, has great oxygen radical absorbance capacity (ORAC) in vitro [40]. Delphinidin has been revealed as the most active scavenger against superoxide anion [41]. Furthermore various reports have shown that anthocyanin has protective effects against oxidative stress in cell lines [42-44]. Several studies have also demonstrated the antioxidant functions of anthocyanins in vivo. Cyanidin-3-glucoside improved oxidative stress-induced hepatic ischemia-reperfusion in rats [45]. Cyanidin, delphinidin, and malvidin induced antioxidant enzymes have been reported to induce upregulation of antioxidant response element (ARE) pathways [46].

Numerous studies have demonstrated the anti-inflammatory effects of anthocyanins as well. Cyanidin-3-glucoside, delphinidin-3-glucoside and petunidin-3-glucoside inhibited NF-kB activities via mitogen activated protein kinase (MAPK) pathways [47-49], and cyanidins inhibited cyclooxygenase enzyme activities [50]. Additionally, cyanidin-3-glucoside had effects on reducing lung inflammation in rats [51].

These biological activities of anthocyanins are closely related to the incidence of chronic diseases. Indeed, anthocyanins reduced risks factors for cardiovascular diseases [17] and suppressed cell growth in various cancer cell lines, indicating that anthocyanins have anticancer properties [52]. Consumption of anthocyanins reduced body weight and insulin resistance, leading to restored glucose tolerance $[53,54]$.

\section{Bioavailability of Anthocyanins}

Most bioactive compounds which are well-known for their great health benefits are generally ingested by food, the study of food as a mixture of its bioactive compounds is more relevant. Supplementation of anthocyanin mixtures reduced inflammatory markers in hypercholesterol subjects rather than individual anthocyanins [18]. Combinations of blackberries and raspberries, for instance, have synergistic antioxidant capacity [55].

Besides the total content of bioactive compounds in food, bioavailability including metabolism and excretion is critical as well. Although anthocyanins have been reported to have low bioavailability after evaluating plasma levels after ingestion of anthocyanin-rich food, recent studies have started to focus on anthocyanin metabolites which were not able to be detected previously, using traditional methods. Interestingly, bioavailability of anthocyanin metabolites was reported to be higher than that of parent anthocyanins by 42-fold [56]. Newly identified anthocyanin metabolites contribute to the improvement of human health [57].

Furthermore, various attempts have been made to improve the bioavailability of flavonoids including anthocyanins using a novel delivery system. Nanoparticles, encapsulation of food, liposomes and gel emersions have been studied to enhance intestinal absorption of food [58]. Encapsulated anthocyanins in liposomes and alginate/chitosan microencapsulation of anthocyanins were valuable for increasing bioefficacy, suggesting application of anthocyanins on nutraceutical development $[59,60]$. 


\section{Studies of Dietary Anthocyanins on Regulation of Inflammation and Obesity in Various Models}

Table 2 presents the beneficial effects of dietary anthocyanins on obesity-related metabolic markers and inflammatory markers in cells and animals. Because there are many studies with respect to the effects of anthocyanins on obesity and inflammation, we look at studies of dietary anthocyanins which are clearly defined for their bioactive compounds on metabolism and inflammation in specific models.

It is difficult to demonstrate anti-inflammatory and anti-adipogenic effects in the same cell line due to each cell's different characteristics. One study reported that purple sweet potato extract exerted antilipogenic activities by suppression of adipogenic enzymes and transcription factors, and had an anti-inflammatory effect by down-regulating COX-2, IL-6 and MCP-1 in 3T3-L1 mouse adipocytes [61].

Red cabbage microgreen decreased weight gain, low density lipoprotein (LDL) levels, triacylglycerol, and cholesterol levels in high fat diet-fed mice. Inflammatory cytokines such as CRP and TNF- $\alpha$ were also significantly diminished in mice [62]. Since blueberries contain a distinct amount of anthocyanins, this has attracted several research studies on their health benefits. High fat diets containing blueberry-fed mice have shown reduced body weight and blood glucose levels as well as TNF- $\alpha$ and IL-6 levels than that of high fat diet-fed control mice [63]. In addition, another study demonstrated that whole blueberry improved high fat diet-induced insulin resistance, and decreased TNF- $\alpha$, IL-6, MCP-1, CD11c+, and inducible nitric oxide synthase (iNOS) [64]. Blueberry juice was also effective in reducing body weight, insulin and leptin levels in serum, cholesterol and triacylglycerol in the liver, and inflammatory markers such as TNF- $\alpha$, IL-6, iNOS, and NF- $\mathrm{kB}$ were decreased in the epididymal adipose tissue of high fat diet-fed mice. This efficacy was also similarly observed in mulberry juice-supplemented obese mice [65]. Other anthocyanin-rich blackcurrant [66], mulberry, cherry [67], black elderberry [68], black soybean [69], freeze-dried jaboticaba peel [70] lowered fat accumulation, triacylglycerol, cholesterol levels in blood serum or liver in high fat diet-fed mice. Rats ingesting fructose rich diets appeared to become obese. However, chokeberry extract was added to the drinking water of the rats, leading to improved metabolic disturbance and inflammation [71]. In addition, tart cherry reduced metabolic and inflammatory factors in Zucker fatty rats [72].

Several clinical studies have also been conducted to evaluate biological functions of dietary anthocyanins on inflammation and obesity, as shown in Table 3. Due to the lack of clinical studies, studies without characterization of anthocyanins were also included in the list. Human subjects whose BMI (body mass index) is over 23 or have a waist circumference over $90 \mathrm{~cm}$ (over $85 \mathrm{~cm}$ in case of female) were allocated to either placebo or black soybean extract-administered groups. This randomized, double-blinded, clinical study showed reduction in abdominal fat, cholesterol, triacylglycerol and LDL levels, with decreasing TNF- $\alpha$ and MCP-1 levels [73]. Red orange juice was also effective in reducing metabolic markers and inflammatory markers in human subjects. 
Table 2. Effects of dietary anthocyanins on obesity and inflammation (cell and animal study).

\begin{tabular}{|c|c|c|c|c|c|c|}
\hline Food Sources & Identified Bioactive Dose of Anthocyanins & Mediators & Inducer & Metabolic Marker & $\begin{array}{c}\text { Inflammatory } \\
\text { Marker }\end{array}$ & Ref. \\
\hline Purple sweet potato & $\begin{array}{l}\text { cyanidnin } \\
\text { (3-caffeylferulysophoroside-5-glucoside) } \\
\text { peonidin } \\
\text { (3-caffeylferulysophoroside-5-glucoside) } \\
\text { Dose: } 4.28 \mu \mathrm{g} / \mathrm{mL} \text { to } 12.84 \mu \mathrm{\mu g} \mathrm{mL}^{\mathrm{a}}\end{array}$ & 3T3-L1 & $\begin{array}{l}\text { Stimuli vs. } \\
\text { undifferentiated cells }\end{array}$ & $\begin{array}{c}\text { leptin } \downarrow \\
\text { adipogenic factors } \downarrow\end{array}$ & $\begin{aligned} \text { COX- } 2 \downarrow \\
\text { MCP- } 1 \downarrow \\
\text { IL-6 } \downarrow\end{aligned}$ & [61] \\
\hline $\begin{array}{l}\text { Red cabbage } \\
\text { microgreen }\end{array}$ & $\begin{array}{l}\text { cyanidin } \\
\text { (3-diglucoside-5-glucoside) } \\
\text { cyanidin } \\
\text { (3-(sinapoyl)-diglucoside-5-glucosides) } \\
\text { cyanidin } \\
\text { (3-(glucosyl)(sinapoyl)(p-coumaroyl)sophorside-5-glucoside) } \\
\text { cyanidin } \\
\text { (3-(glucosyl)(sinapoyl)(feruloyl)sophorside-5-glucoside) } \\
\text { cyanidin } \\
\text { (3-diferuloylsophoroside-5-glucoside) } \\
\text { cyanidin } \\
\text { (3-(coumaroyl)sophoroside-5-glucoside) } \\
\text { cyanidin } \\
\text { (3-(feruloyl)sophoroside-5-glucoside) } \\
\text { cyanidin } \\
\text { (3-diferuloylsophoroside-5-glucoside) } \\
\text { cyanidin } \\
\text { (3-(sinapoyl)(feruloyl)sophoroside-5-glucoside) } \\
\text { cyanidin } \\
\text { (3-(sinapoyl)(sinapoyl)sophoroside-5-glucoside) } \\
\text { Dose: } 139.596 \text { nmol/gb }\end{array}$ & mice (C57BL/6) & $\begin{array}{l}\text { high fat-diet vs. } \\
\text { normal-diet for } 8 \text { weeks }\end{array}$ & $\begin{array}{c}\text { LDL } \downarrow \\
\text { cholesterol } \downarrow \\
\text { TG } \downarrow\end{array}$ & $\begin{array}{c}\mathrm{CRP} \downarrow \\
\mathrm{TNF}-\alpha \downarrow\end{array}$ & [62] \\
\hline Blueberry & $\begin{array}{l}\text { delphinidins } \\
\text { cyanidins } \\
\text { peonidins } \\
\text { malvidins } \\
\text { Dose: } 1.29 \mathrm{mg} / \mathrm{g}^{\mathrm{b}}\end{array}$ & mice (C57BL/6) & $\begin{array}{l}\text { high fat-diet vs. normal } \\
\text { diet for } 8 \text { weeks }\end{array}$ & glucose $\downarrow$ & $\begin{array}{c}\text { TNF- } \alpha \downarrow \text { IL-6 } \downarrow \\
\text { MCP- } 1 \downarrow \text { iNOS } \downarrow \\
\text { IL-10 } \uparrow \text { CD11c }+\end{array}$ & [64] \\
\hline
\end{tabular}


Table 2. Cont

\begin{tabular}{|c|c|c|c|c|c|c|}
\hline Food Sources & Identified Bioactive Dose of Anthocyanins & Mediators & Inducer & Metabolic Marker & $\begin{array}{c}\text { Inflammatory } \\
\text { Marker }\end{array}$ & Ref. \\
\hline Blueberry & $\begin{array}{l}\text { cyanidin } \\
\text { (3-galactoside) } \\
\text { cyanidin } \\
\text { (3-arabinoside) } \\
\text { delphinidin } \\
\text { (3-arabinoside) } \\
\text { delphinidin } \\
\text { (3-galactoside) } \\
\text { petunidin } \\
\text { (3-glucoside) } \\
\text { petunidin } \\
\text { (3-arabinoside) } \\
\text { malvidin } \\
\text { (3-galactoside) } \\
\text { malvidin } \\
\text { (3-glucoside) } \\
\text { Dose: } 50 \text { to } 200 \mu \mathrm{g} / \mathrm{g} \text { b }\end{array}$ & mice (C57BL/6) & $\begin{array}{l}\text { high fat-diet vs. normal } \\
\text { diet for } 8 \text { weeks }\end{array}$ & $\begin{array}{c}\text { glucose } \downarrow \\
\text { TG } \downarrow \\
\text { cholesterol } \downarrow \\
\text { insulin } \downarrow \\
\text { leptin } \downarrow\end{array}$ & $\begin{array}{c}\text { TNF- } \alpha \downarrow \\
\text { IL-6 } \downarrow\end{array}$ & [63] \\
\hline Black elderberry & $\begin{array}{l}\text { cyanidin } \\
\text { (3-glucoside) } \\
\text { cyanidin } \\
\text { (3-sambubioside) } \\
\text { Dose: } 3.334,1.7 \mu \mathrm{g} / \mathrm{g}\end{array}$ & mice (C57BL/6) & $\begin{array}{l}\text { high fat-diet vs. normal } \\
\text { diet for } 16 \text { weeks }\end{array}$ & $\begin{array}{c}\text { TG } \downarrow \\
\text { insulin } \downarrow \\
\text { cholesterol } \downarrow\end{array}$ & $\begin{array}{l}\text { MCP- } 1 \downarrow \\
\text { TNF- } \alpha \downarrow\end{array}$ & [68] \\
\hline Blackcurrant & $\begin{array}{l}\text { delphinidin } \\
\text { (3-glucoside) } \\
\text { delphinidin } \\
\text { (3-o-rutinoside) } \\
\text { cyanidin } \\
\text { (3-glucoside) } \\
\text { cyanidin } \\
\text { (3-rutinoside) } \\
\text { Dose: } 298.1 \mu \mathrm{g} / \mathrm{g} \text { b } \\
\end{array}$ & mice (C57BL/6) & $\begin{array}{c}\text { high } \\
\text { fat/cholesterol-diet vs. } \\
\text { normal diet for } 12 \\
\text { weeks }\end{array}$ & adipogenic genes $\downarrow$ & $\begin{array}{c}\text { TNF- } \alpha \downarrow \\
\text { IL-6 } \downarrow \\
\text { IL-1 } \beta \downarrow\end{array}$ & [66] \\
\hline Mulberry & $\begin{array}{l}\text { cyanidin } \\
\text { (3-glucoside) } \\
\text { cyanidin } \\
\text { (3-rutinoside) } \\
\text { pelarginidin } \\
\text { (3-glucose) } \\
\text { Dose: } 200 \mu \mathrm{gg} / \mathrm{g}\end{array}$ & mice (C57BL/6) & $\begin{array}{l}\text { high fat-diet vs. normal } \\
\text { diet for } 16 \text { weeks }\end{array}$ & $\begin{array}{c}\text { glucose } \downarrow \\
\text { leptin } \downarrow\end{array}$ & $\begin{array}{c}\text { TNF- } \alpha \downarrow \\
\text { IL-6 } \downarrow \\
\text { iNOS } \downarrow \\
\text { NF-kB } \downarrow\end{array}$ & [67] \\
\hline
\end{tabular}


Table 2. Cont

\begin{tabular}{|c|c|c|c|c|c|c|}
\hline Food Sources & Identified Bioactive Dose of Anthocyanins & Mediators & Inducer & Metabolic Marker & $\begin{array}{c}\text { Inflammatory } \\
\text { Marker }\end{array}$ & Ref. \\
\hline Cherry & $\begin{array}{l}\text { cyanidin } \\
\text { (3-2G-glucosylrutinoside) } \\
\text { cyanidin } \\
\text { (3-rutinoside) } \\
\text { pelarginidin } \\
\text { (3-rutinoside) } \\
\text { Dose: } 200 \mu \mathrm{g} / \mathrm{g}^{\mathrm{b}}\end{array}$ & mice (C57BL/6) & $\begin{array}{l}\text { high fat-diet vs. normal } \\
\text { diet for } 16 \text { weeks }\end{array}$ & $\begin{array}{c}\text { glucose } \downarrow \\
\text { leptin } \downarrow\end{array}$ & $\begin{array}{c}\text { TNF- } \alpha \downarrow \\
\text { IL-6 } \downarrow \\
\text { iNOS } \downarrow \\
\text { NF-kB } \downarrow\end{array}$ & [67] \\
\hline Blueberry juice & $\begin{array}{l}\text { cyanidin } \\
\text { (3-galactoside) } \\
\text { cyanidin } \\
\text { (3-arabinoside) } \\
\text { delphinidin } \\
\text { (3-glucoside) } \\
\text { delphinidin } \\
\text { (3-galactoside) } \\
\text { delphinidin } \\
\text { (3-arabinoside) } \\
\text { petunidin } \\
\text { (3-glucoside) } \\
\text { petunidin } \\
\text { (3-arabinoside) } \\
\text { malvidin } \\
\text { (3-galactoside) } \\
\text { malvidin } \\
\text { (3-glucoside) } \\
\text { Dose: } 4.09 \mathrm{mg} / \mathrm{mL}^{\mathrm{c}} \\
\end{array}$ & mice (C57BL/6) & $\begin{array}{l}\text { high fat-diet vs. normal } \\
\text { diet for } 12 \text { weeks }\end{array}$ & $\begin{array}{c}\text { leptin } \downarrow \\
\text { cholesterol } \downarrow \\
\text { adiponectin } \uparrow \\
\text { TG } \downarrow\end{array}$ & $\begin{array}{c}\text { TNF- } \alpha \downarrow \\
\text { IL-6 } \downarrow\end{array}$ & [65] \\
\hline Mulberry juice & $\begin{array}{l}\text { cyanidin } \\
\text { (3-glucoside) } \\
\text { cyanidin } \\
\text { (3-rutinoside) } \\
\text { pelargonidin } \\
\text { (3-glucoside) } \\
\text { pelargonidin } \\
\text { (3-rutinoside) } \\
\text { Dose: } 21.86 \mathrm{mg} / \mathrm{mL}^{\mathrm{c}}\end{array}$ & mice (C57BL/6) & $\begin{array}{l}\text { high fat-diet vs. normal } \\
\text { diet for } 12 \text { weeks }\end{array}$ & $\begin{array}{c}\text { leptin } \downarrow \\
\text { adiponectin } \uparrow\end{array}$ & $\begin{array}{c}\text { TNF- } \alpha \downarrow \\
\text { IL-6 } \downarrow \downarrow\end{array}$ & [65] \\
\hline
\end{tabular}


Table 2. Cont

\begin{tabular}{|c|c|c|c|c|c|c|}
\hline Food Sources & Identified Bioactive Dose of Anthocyanins & Mediators & Inducer & Metabolic Marker & $\begin{array}{l}\text { Inflammatory } \\
\text { Marker }\end{array}$ & Ref. \\
\hline Black soybean & $\begin{array}{l}\text { delphinidin } \\
\text { (3-glucoside) } \\
\text { cyanidin } \\
\text { (3-glucoside) } \\
\text { petunidin } \\
\text { (3-glucoside) } \\
\text { pelargonodin } \\
\text { (3-glucoside) } \\
\text { peonidin } \\
\text { (3-glucoside) } \\
\text { Dose: } 12.48 \mathrm{mg} / \mathrm{g} \text { b }\end{array}$ & mice (C57BL/6) & $\begin{array}{l}\text { high fat-diet vs. normal } \\
\text { diet for } 12 \text { weeks }\end{array}$ & $\begin{array}{c}\text { TG } \downarrow \\
\text { cholesterol } \downarrow\end{array}$ & $\begin{array}{c}\text { TNF- } \alpha \downarrow \\
\text { IL-6 } \downarrow \\
\text { IL-10^ }\end{array}$ & [69] \\
\hline Jaboticaba peel & $\begin{array}{l}\text { delphinidin } \\
\text { (3- O-glycoside) } \\
\text { cyanidin } \\
\text { (3- } O \text {-glycoside) } \\
\text { Dose: } 259.9,519.8,1039.6 \mu \mathrm{g} / \mathrm{g}\end{array}$ & swiss inbred mice & $\begin{array}{l}\text { high fat-diet vs. normal } \\
\text { diet for } 6 \text { weeks }\end{array}$ & insulin $\downarrow$ & $\begin{array}{l}\text { IL-6 } 6 \downarrow \\
\text { IL-1 } \beta \downarrow\end{array}$ & {$[70]$} \\
\hline Chokeberry & $\begin{array}{l}\text { total anthocyanin } \\
\text { Dose: } 10 \text { or } 20 \mathrm{mg} / \mathrm{kg}\end{array}$ & Wistar rat & $\begin{array}{l}\text { fructose-rich diet vs. } \\
\text { normal diet for } 6 \text { weeks }\end{array}$ & $\begin{array}{c}\text { glucose } \downarrow \text { insulin } \downarrow \text { TG } \downarrow \\
\text { cholesterol } \downarrow\end{array}$ & $\begin{array}{c}\text { TNF- } \alpha \downarrow \\
\text { IL-6 } \downarrow\end{array}$ & [71] \\
\hline Tart cherry & $\begin{array}{l}\text { cyanidin } \\
\text { (3-sophoroside) } \\
\text { cyanidin } \\
\text { (3-glucosylrutinoside) } \\
\text { cyanidin- } \\
\text { (3-glucoside) } \\
\text { cyanidin } \\
\text { (3-rutinoside) } \\
\text { peonidin } \\
\text { (3-glucoside) } \\
\text { Pelargonidin } \\
\text { Dose: } 0.6598 \mathrm{mg} / \mathrm{g} \text { b }\end{array}$ & zucker fatty rats & $\begin{array}{l}\text { Spontaneously obese } \\
\text { for } 90 \text { days }\end{array}$ & $\begin{array}{c}\text { glucose } \downarrow \\
\text { insulin } \downarrow \\
\text { cholesterol } \downarrow \\
\quad \text { TG } \downarrow\end{array}$ & $\begin{array}{c}\text { TNF- } \alpha \downarrow \\
\text { IL- } 6 \downarrow\end{array}$ & [72] \\
\hline
\end{tabular}

weight. CRP: C-reactive protein. 
Table 3. Effects of dietary anthocyanins on obesity and inflammation (clinical study).

\begin{tabular}{|c|c|c|c|c|c|}
\hline Food Sources & $\begin{array}{l}\text { Bioactives Dose of } \\
\text { Anthocyanins }\end{array}$ & Subject Duration & $\begin{array}{l}\text { Metabolic } \\
\text { Marker }\end{array}$ & $\begin{array}{l}\text { Inflammatory } \\
\text { Marker }\end{array}$ & Ref. \\
\hline Black soybean & $\begin{array}{c}\text { cyanidin } \\
\text { (3-glucosides) } \\
\text { delphinidin } \\
\text { (3-glucoside) } \\
\text { petunidin } \\
\text { (3-glucoside) } \\
\text { 31.48 mg/day }\end{array}$ & $\begin{array}{c}\text { BMI }>23 \\
\text { WC }>90 \text { for male } \\
\text { WC }>85 \text { for female } \\
\text { For } 8 \text { weeks }\end{array}$ & $\begin{array}{c}\text { TG } \downarrow \\
\text { cholesterol } \downarrow \\
\text { LDL } \downarrow\end{array}$ & $\begin{array}{l}\text { TNF- } \alpha \downarrow \\
\text { MCP- } 1 \downarrow\end{array}$ & [73] \\
\hline Red orange juice & $\begin{array}{l}\text { Anthocyanin mixture } \\
250 \mathrm{mg} / \text { day }\end{array}$ & $\begin{array}{l}\text { average BMI }=34.4 \pm 4.8 \\
\text { for } 12 \text { weeks }\end{array}$ & $\begin{array}{c}\Delta \text { leptin } \downarrow \\
\Delta \text { adiponectin } \downarrow\end{array}$ & $\begin{array}{c}\text { CRP } \downarrow \\
\text { TNF- } \alpha \downarrow\end{array}$ & [74] \\
\hline $\begin{array}{c}\text { Red-fleshed sweet } \\
\text { orange juice }\end{array}$ & $\begin{array}{c}\text { anthocyanin mixture } \\
750 \mathrm{~mL} / \text { day }\end{array}$ & $\begin{array}{c}\text { age } 23-59 \\
\text { BMI } 18.5-24.986 .4 / 74.6\end{array}$ & cholesterol $\downarrow$ & $\mathrm{CRP} \downarrow$ & [75] \\
\hline
\end{tabular}

BMI: body mass index; WC: waist circumstance; CRP: C-reactive protein; $\Delta$ : incremental change.

\section{Summary}

Previously, obesity was regarded as just a matter of excessive energy storage triggered by energy imbalance, however many studies have indicated that chronic low-grade inflammation in adipose tissue can be an important issue in obesity. In particular, this metainflammation could enable pathogenesis of chronic diseases associated with obesity. Anthocyanins have great biological activities and low toxicity in vivo, therefore many scientists are interested in the health benefits of anthocyanins, as well as their application in preventing and treating chronic diseases, including obesity. Here, we describe the positive effects of dietary anthocyanins limited to their well-defined components against metabolic and inflammatory markers in cell, animal and human obesity models. In addition, anthocyanin mixtures found in food such as red cabbage microgreen, blueberry, blackcurrant, mulberry, cherry, black elderberry, black soybean, chokeberry and jaboticaba peel (in whole or extract) interestingly had higher clinical efficacy than single anthocyanins.

\section{Conclusions}

We reviewed the effects of anthocyanins-rich food on attenuating obesity and inflammation in cells, animals, and humans. Taken together, dietary anthocyanins may be a potential regulator of obesity-derived inflammation and its associated chronic diseases, as presented in Figure 2.

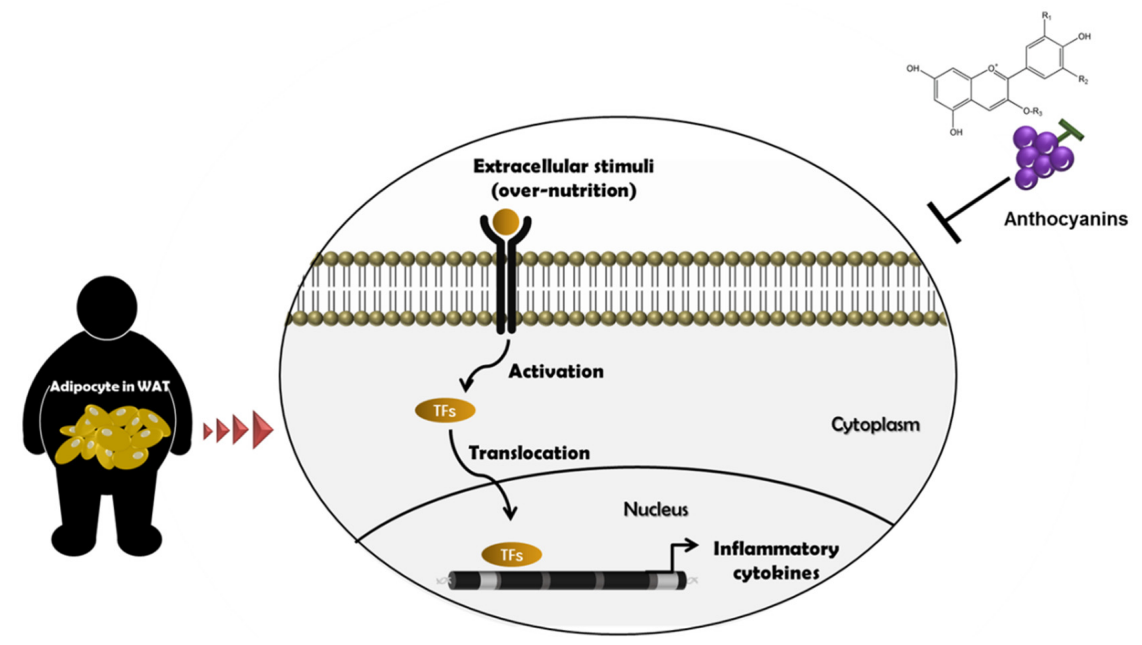

Figure 2. Beneficial effects of anthocyanins on obesity and inflammation. When receptors recognize the status of over-nutrition, they activate various transcription factors such as NF- $\mathrm{B}$, IRF-3 and AP-1 for translocation into nucleus and bind to the promoter region of target genes. Eventually, inflammatory cytokines are expressed resulting in chronic inflammatory conditions in adipocytes of WAT. Dietary anthocyanins may have preventive effects on these events, leading to health benefits. 
Acknowledgments: Supported by Rural Development Administration, Republic of Korea (PJ011885).

Author Contributions: Y.M. Lee, and K.J. Yeum conceived and wrote the manuscript. And Y. Yoon, H. Yoon, and H.M. Park designed the table. S. Song developed the figure design.

Conflicts of Interest: The authors declare no conflict of interest.

\section{Abbreviation}

$\begin{array}{ll}\text { TG } & \text { triacylglycerol } \\ \text { LDL } & \text { low density lipoprotein } \\ \text { COX-2 } & \text { cyclooxygenase-2 } \\ \text { TNF- } \alpha & \text { tumor necrosis factor- } \alpha \\ \text { IL-6 } & \text { interleukin-6 } \\ \text { IL-1 } \beta & \text { interleukin-1 } \beta \\ \text { IL-10 } & \text { interleukin-10 } \\ \text { iNOS } & \text { inducible nitric synthase } \\ \text { MCP-1 } & \text { monocyte chemoattactant protein-1 } \\ \text { NF-KB } & \text { nuclear factor kappa B } \\ \text { BMI } & \text { body mass index } \\ \text { CRP } & \text { c-reactive protein } \\ \text { WC } & \text { waist circumstance } \\ \text { IRF-3 } & \text { interferon regulatory factor 3 } \\ \text { AP-1 } & \text { activated protein-1 } \\ \text { WAT } & \text { white adipose tissue }\end{array}$

\section{References}

1. Adams, K.F.; Schatzkin, A.; Harris, T.B.; Kipnis, V.; Mouw, T.; Ballard-Barbash, R.; Hollenbeck, A.; Leitzmann, M.F. Overweight, obesity, and mortality in a large prospective cohort of persons 50 to 71 years old. N. Engl. J. Med. 2006, 355, 763-778. [CrossRef] [PubMed]

2. Finucane, M.M.; Stevens, G.A.; Cowan, M.J.; Danaei, G.; Lin, J.K.; Paciorek, C.J.; Singh, G.M.; Gutierrez, H.R.; Lu, Y.; Bahalim, A.N.; et al. National, regional, and global trends in body-mass index since 1980: Systematic analysis of health examination surveys and epidemiological studies with 960 country-years and 9.1 million participants. Lancet 2011, 377, 557-567. [CrossRef]

3. Greenberg, A.S.; Obin, M.S. Obesity and the role of adipose tissue in inflammation and metabolism. Am. J. Clin. Nutr. 2006, 83, S461-S465.

4. Osborn, O.; Olefsky, J.M. The cellular and signaling networks linking the immune system and metabolism in disease. Nat. Med. 2012, 18, 363-374. [CrossRef] [PubMed]

5. Gregor, M.F.; Hotamisligil, G.S. Inflammatory mechanisms in obesity. Annu. Rev. Immunol. 2011, $29,415-445$. [CrossRef] [PubMed]

6. Mraz, M.; Haluzik, M. The role of adipose tissue immune cells in obesity and low-grade inflammation. J. Endocrinol. 2014, 222, R113-R127. [CrossRef] [PubMed]

7. Brooks-Worrell, B.; Narla, R.; Palmer, J.P. Biomarkers and immune-modulating therapies for type 2 diabetes. Trends Immunol. 2012, 33, 546-553. [CrossRef] [PubMed]

8. Reilly, S.M.; Chiang, S.H.; Decker, S.J.; Chang, L.; Uhm, M.; Larsen, M.J.; Rubin, J.R.; Mowers, J.; White, N.M.; Hochberg, I.; et al. An inhibitor of the protein kinases tbk1 and ikk-varepsilon improves obesity-related metabolic dysfunctions in mice. Nat. Med. 2013, 19, 313-321. [CrossRef] [PubMed]

9. Garrow, J.S. Magic bullets now uncontrolled. BMJ 2010, 340, c1351. [CrossRef] [PubMed]

10. Cefalu, W.T.; Ye, J.; Zuberi, A.; Ribnicky, D.M.; Raskin, I.; Liu, Z.; Wang, Z.Q.; Brantley, P.J.; Howard, L.; Lefevre, M. Botanicals and the metabolic syndrome. Am. J. Clin. Nutr. 2008, 87, S481-S487.

11. Czemplik, M.; Kulma, A.; Wang, Y.F.; Szopa, J. Therapeutic strategies of plant-derived compounds for diabetes via regulation of monocyte chemoattractant protein-1. Curr. Med. Chem. 2017, 24, 1453-1468. [CrossRef] [PubMed] 
12. He, J.; Giusti, M.M. Anthocyanins: Natural colorants with health-promoting properties. Annu. Rev. Food Sci. Technol. 2010, 1, 163-187. [CrossRef] [PubMed]

13. Barzilay, J.I.; Abraham, L.; Heckbert, S.R.; Cushman, M.; Kuller, L.H.; Resnick, H.E.; Tracy, R.P. The relation of markers of inflammation to the development of glucose disorders in the elderly: The cardiovascular health study. Diabetes 2001, 50, 2384-2389. [CrossRef] [PubMed]

14. Mazza, G.; Miniati, E. Anthocyanins in Fruits, Vegetables and Grains, 1st ed.; CRC Press: Boca Raton, FL, USA, 1993.

15. De Pascual-Teresa, S.; Sanchez-Ballesta, M.T. Anthocyanins: From plant to health. Phytochem. Rev. 2008, 7, 281-299. [CrossRef]

16. Valenti, L.; Riso, P.; Mazzocchi, A.; Porrini, M.; Fargion, S.; Agostoni, C. Dietary anthocyanins as nutritional therapy for nonalcoholic fatty liver disease. Oxid. Med. Cell. Longev. 2013, 2013. [CrossRef] [PubMed]

17. Wallace, T.C.; Slavin, M.; Frankenfeld, C.L. Systematic review of anthocyanins and markers of cardiovascular disease. Nutrients 2016, 8, 32. [CrossRef] [PubMed]

18. Zhu, Y.; Ling, W.; Guo, H.; Song, F.; Ye, Q.; Zou, T.; Li, D.; Zhang, Y.; Li, G.; Xiao, Y.; et al. Anti-inflammatory effect of purified dietary anthocyanin in adults with hypercholesterolemia: A randomized controlled trial. Nutr. Metab. Cardiovasc. Dis. 2013, 23, 843-849. [CrossRef] [PubMed]

19. Bastard, J.P.; Maachi, M.; Lagathu, C.; Kim, M.J.; Caron, M.; Vidal, H.; Capeau, J.; Feve, B. Recent advances in the relationship between obesity, inflammation, and insulin resistance. Eur. Cytokine Netw. 2006, 17, 4-12. [PubMed]

20. Lumeng, C.N.; Saltiel, A.R. Inflammatory links between obesity and metabolic disease. J. Clin. Investig. 2011, 121, 2111-2117. [CrossRef] [PubMed]

21. Ford, E.S. The metabolic syndrome and c-reactive protein, fibrinogen, and leukocyte count: Findings from the third national health and nutrition examination survey. Atherosclerosis 2003, 168, 351-358. [CrossRef]

22. Odegaard, J.I.; Chawla, A. The immune system as a sensor of the metabolic state. Immunity 2013, 38, 644-654. [CrossRef] [PubMed]

23. Kumari, M.; Wang, X.; Lantier, L.; Lyubetskaya, A.; Eguchi, J.; Kang, S.; Tenen, D.; Roh, H.C.; Kong, X.; Kazak, L.; et al. Irf3 promotes adipose inflammation and insulin resistance and represses browning. J. Clin. Investig. 2016, 126, 2839-2854. [CrossRef] [PubMed]

24. Ali, F.; Ismail, A.; Kersten, S. Molecular mechanisms underlying the potential antiobesity-related diseases effect of cocoa polyphenols. Mol. Nutr. Food Res. 2014, 58, 33-48. [CrossRef] [PubMed]

25. O'Neill, L.A.; Hardie, D.G. Metabolism of inflammation limited by ampk and pseudo-starvation. Nature 2013, 493, 346-355. [CrossRef] [PubMed]

26. Wang, Q.A.; Scherer, P.E.; Gupta, R.K. Improved methodologies for the study of adipose biology: Insights gained and opportunities ahead. J. Lipid Res. 2014, 55, 605-624. [CrossRef] [PubMed]

27. Ruiz-Ojeda, F.J.; Ruperez, A.I.; Gomez-Llorente, C.; Gil, A.; Aguilera, C.M. Cell models and their application for studying adipogenic differentiation in relation to obesity: A review. Int. J. Mol. Sci. 2016, 17, 1040. [CrossRef] [PubMed]

28. Moseti, D.; Regassa, A.; Kim, W.K. Molecular regulation of adipogenesis and potential anti-adipogenic bioactive molecules. Int. J. Mol. Sci. 2016, 17, 124. [CrossRef] [PubMed]

29. Esteve Rafols, M. Adipose tissue: Cell heterogeneity and functional diversity. Endocrinol. Nutr. 2014, 61, 100-112. [CrossRef] [PubMed]

30. Cawthorn, W.P.; Scheller, E.L.; MacDougald, O.A. Adipose tissue stem cells meet preadipocyte commitment: Going back to the future. J. Lipid Res. 2012, 53, 227-246. [CrossRef] [PubMed]

31. Armani, A.; Mammi, C.; Marzolla, V.; Calanchini, M.; Antelmi, A.; Rosano, G.M.; Fabbri, A.; Caprio, M. Cellular models for understanding adipogenesis, adipose dysfunction, and obesity. J. Cell. Biochem. 2010, 110, 564-572. [CrossRef] [PubMed]

32. Lutz, T.A.; Woods, S.C. Overview of animal models of obesity. Curr. Protoc. Pharmacol. 2012. [CrossRef]

33. Zhang, Y.; Proenca, R.; Maffei, M.; Barone, M.; Leopold, L.; Friedman, J.M. Positional cloning of the mouse obese gene and its human homologue. Nature 1994, 372, 425-432. [CrossRef] [PubMed]

34. Frederich, R.C.; Lollmann, B.; Hamann, A.; Napolitano-Rosen, A.; Kahn, B.B.; Lowell, B.B.; Flier, J.S. Expression of ob mrna and its encoded protein in rodents. Impact of nutrition and obesity. J. Clin. Investig. 1995, 96, 1658-1663. [CrossRef] [PubMed] 
35. Phillips, M.S.; Liu, Q.; Hammond, H.A.; Dugan, V.; Hey, P.J.; Caskey, C.J.; Hess, J.F. Leptin receptor missense mutation in the fatty zucker rat. Nat. Genet. 1996, 13, 18-19. [CrossRef] [PubMed]

36. Nilsson, C.; Raun, K.; Yan, F.F.; Larsen, M.O.; Tang-Christensen, M. Laboratory animals as surrogate models of human obesity. Acta Pharmacol. Sin. 2012, 33, 173-181. [CrossRef] [PubMed]

37. Surwit, R.S.; Feinglos, M.N.; Rodin, J.; Sutherland, A.; Petro, A.E.; Opara, E.C.; Kuhn, C.M.; Rebuffe-Scrive, M. Differential effects of fat and sucrose on the development of obesity and diabetes in $\mathrm{c} 57 \mathrm{bl} / 6 \mathrm{j}$ and a/j mice. Metabolism 1995, 44, 645-651. [CrossRef]

38. Schemmel, R.; Mickelsen, O.; Motawi, K. Conversion of dietary to body energy in rats as affected by strain, sex and ration. J. Nutr. 1972, 102, 1187-1197. [PubMed]

39. Levin, B.E.; Hogan, S.; Sullivan, A.C. Initiation and perpetuation of obesity and obesity resistance in rats. Am. J. Physiol. 1989, 256, R766-R771. [PubMed]

40. Wang, H.; Cao, G.; Prior, R.L. Oxygen radical absorbing capacity of anthocyanins. J. Agric. Food Chem. 1997, 45, 304-309. [CrossRef]

41. Tsuda, T.; Shiga, K.; Ohshima, K.; Kawakishi, S.; Osawa, T. Inhibition of lipid peroxidation and the active oxygen radical scavenging effect of anthocyanin pigments isolated from Phaseolus vulgaris L. Biochem. Pharmacol. 1996, 52, 1033-1039. [CrossRef]

42. Tedesco, I.; Luigi Russo, G.; Nazzaro, F.; Russo, M.; Palumbo, R. Antioxidant effect of red wine anthocyanins in normal and catalase-inactive human erythrocytes. J. Nutr. Biochem. 2001, 12, 505-511. [CrossRef]

43. Isaak, C.K.; Petkau, J.C.; Blewett, H.; Karmin, O.; Siow, Y.L. Lingonberry anthocyanins protect cardiac cells from oxidative-stress-induced apoptosis. Can. J. Physiol. Pharmacol. 2017, 95, 904-910. [CrossRef] [PubMed]

44. Heo, H.J.; Lee, C.Y. Strawberry and its anthocyanins reduce oxidative stress-induced apoptosis in pc12 cells. J. Agric. Food Chem. 2005, 53, 1984-1989. [CrossRef] [PubMed]

45. Tsuda, T.; Horio, F.; Osawa, T. The role of anthocyanins as an antioxidant under oxidative stress in rats. Biofactors 2000, 13, 133-139. [CrossRef] [PubMed]

46. Thoppil, R.J.; Bhatia, D.; Barnes, K.F.; Haznagy-Radnai, E.; Hohmann, J.; Darvesh, A.S.; Bishayee, A. Black currant anthocyanins abrogate oxidative stress through nrf2-mediated antioxidant mechanisms in a rat model of hepatocellular carcinoma. Curr. Cancer Drug Targets 2012, 12, 1244-1257. [PubMed]

47. Afaq, F.; Saleem, M.; Krueger, C.G.; Reed, J.D.; Mukhtar, H. Anthocyanin- and hydrolyzable tannin-rich pomegranate fruit extract modulates mapk and nf-kappab pathways and inhibits skin tumorigenesis in cd-1 mice. Int. J. Cancer 2005, 113, 423-433. [CrossRef] [PubMed]

48. Jeong, J.W.; Lee, W.S.; Shin, S.C.; Kim, G.Y.; Choi, B.T.; Choi, Y.H. Anthocyanins downregulate lipopolysaccharide-induced inflammatory responses in bv2 microglial cells by suppressing the nf-kappab and akt/mapks signaling pathways. Int. J. Mol. Sci. 2013, 14, 1502-1515. [CrossRef] [PubMed]

49. Vendrame, S.; Klimis-Zacas, D. Anti-inflammatory effect of anthocyanins via modulation of nuclear factor-kappab and mitogen-activated protein kinase signaling cascades. Nutr. Rev. 2015, 73, 348-358. [CrossRef] [PubMed]

50. Wang, H.; Nair, M.G.; Strasburg, G.M.; Chang, Y.C.; Booren, A.M.; Gray, J.I.; DeWitt, D.L. Antioxidant and antiinflammatory activities of anthocyanins and their aglycon, cyanidin, from tart cherries. J. Nat. Prod. 1999, 62, 294-296. [CrossRef] [PubMed]

51. Rossi, A.; Serraino, I.; Dugo, P.; Di Paola, R.; Mondello, L.; Genovese, T.; Morabito, D.; Dugo, G.; Sautebin, L.; Caputi, A.P.; et al. Protective effects of anthocyanins from blackberry in a rat model of acute lung inflammation. Free Radic. Res. 2003, 37, 891-900. [CrossRef] [PubMed]

52. Lin, B.W.; Gong, C.C.; Song, H.F.; Cui, Y.Y. Effects of anthocyanins on the prevention and treatment of cancer. Br. J. Pharmacol. 2017, 174, 1226-1243. [CrossRef] [PubMed]

53. Vendrame, S.; Del Bo, C.; Ciappellano, S.; Riso, P.; Klimis-Zacas, D. Berry fruit consumption and metabolic syndrome. Antioxidants 2016, 5, 34. [CrossRef] [PubMed]

54. Overall, J.; Bonney, S.A.; Wilson, M.; Beermann, A.; Grace, M.H.; Esposito, D.; Lila, M.A.; Komarnytsky, S. Metabolic effects of berries with structurally diverse anthocyanins. Int. J. Mol. Sci. 2017, 18, 422. [CrossRef] [PubMed]

55. Wang, S.; Meckling, K.A.; Marcone, M.F.; Kakuda, Y.; Tsao, R. Synergistic, additive, and antagonistic effects of food mixtures on total antioxidant capacities. J. Agric. Food Chem. 2011, 59, 960-968. [CrossRef] [PubMed] 
56. Czank, C.; Cassidy, A.; Zhang, Q.; Morrison, D.J.; Preston, T.; Kroon, P.A.; Botting, N.P.; Kay, C.D. Human metabolism and elimination of the anthocyanin, cyanidin-3-glucoside: A (13)c-tracer study. Am. J. Clin. Nutr. 2013, 97, 995-1003. [CrossRef] [PubMed]

57. Manach, C.; Williamson, G.; Morand, C.; Scalbert, A.; Remesy, C. Bioavailability and bioefficacy of polyphenols in humans. I. Review of 97 bioavailability studies. Am. J. Clin. Nutr. 2005, 81, S230-S242.

58. Rein, M.J.; Renouf, M.; Cruz-Hernandez, C.; Actis-Goretta, L.; Thakkar, S.K.; da Silva Pinto, M. Bioavailability of bioactive food compounds: A challenging journey to bioefficacy. Br. J. Clin. Pharmacol. 2013, 75, 588-602. [CrossRef] [PubMed]

59. Zhao, L.; Temelli, F.; Chen, L. Encapsulation of anthocyanin in liposomes using supercritical carbon dioxide: Effects of anthocyanin and sterol concentrations. J. Func. Food 2017, 34, 159-167. [CrossRef]

60. Kanokpanont, S.; Yamdech, R.; Aramwit, P. Stability enhancement of mulberry-extracted anthocyanin using alginate/chitosan microencapsulation for food supplement application. Artif. Cells Nanomed. Biotechnol. 2017. [CrossRef] [PubMed]

61. Ju, J.H.; Yoon, H.S.; Park, H.J.; Kim, M.Y.; Shin, H.K.; Park, K.Y.; Yang, J.O.; Sohn, M.S.; Do, M.S. Anti-obesity and antioxidative effects of purple sweet potato extract in 3t3-11 adipocytes in vitro. J. Med. Food 2011, 14, 1097-1106. [CrossRef] [PubMed]

62. Huang, H.; Jiang, X.; Xiao, Z.; Yu, L.; Pham, Q.; Sun, J.; Chen, P.; Yokoyama, W.; Yu, L.L.; Luo, Y.S.; et al. Red cabbage microgreens lower circulating low-density lipoprotein (ldl), liver cholesterol, and inflammatory cytokines in mice fed a high-fat diet. J. Agric. Food Chem. 2016, 64, 9161-9171. [CrossRef] [PubMed]

63. Wu, T.; Jiang, Z.; Yin, J.; Long, H.; Zheng, X. Anti-obesity effects of artificial planting blueberry (vaccinium ashei) anthocyanin in high-fat diet-treated mice. Int. J. Food Sci. Nutr. 2016, 67, 257-264. [CrossRef] [PubMed]

64. DeFuria, J.; Bennett, G.; Strissel, K.J.; Perfield, J.W., 2nd; Milbury, P.E.; Greenberg, A.S.; Obin, M.S. Dietary blueberry attenuates whole-body insulin resistance in high fat-fed mice by reducing adipocyte death and its inflammatory sequelae. J. Nutr. 2009, 139, 1510-1516. [CrossRef] [PubMed]

65. Wu, T.; Tang, Q.; Gao, Z.; Yu, Z.; Song, H.; Zheng, X.; Chen, W. Blueberry and mulberry juice prevent obesity development in c57bl/ 6 mice. PLoS ONE 2013, 8, e77585. [CrossRef] [PubMed]

66. Benn, T.; Kim, B.; Park, Y.K.; Wegner, C.J.; Harness, E.; Nam, T.G.; Kim, D.O.; Lee, J.S.; Lee, J.Y. Polyphenol-rich blackcurrant extract prevents inflammation in diet-induced obese mice. J. Nutr. Biochem. 2014, 25, 1019-1025. [CrossRef] [PubMed]

67. Wu, T.; Yin, J.; Zhang, G.; Long, H.; Zheng, X. Mulberry and cherry anthocyanin consumption prevents oxidative stress and inflammation in diet-induced obese mice. Mol. Nutr. Food Res. 2016, 60, 687-694. [CrossRef] [PubMed]

68. Farrell, N.J.; Norris, G.H.; Ryan, J.; Porter, C.M.; Jiang, C.; Blesso, C.N. Black elderberry extract attenuates inflammation and metabolic dysfunction in diet-induced obese mice. Br. J. Nutr. 2015, 114, 1123-1131. [CrossRef] [PubMed]

69. Kim, S.Y.; Wi, H.-R.; Choi, S.; Ha, T.J.; Lee, B.W.; Lee, M. Inhibitory effect of anthocyanin-rich black soybean testa (Glycine $\max$ (L.) merr.) on the inflammation-induced adipogenesis in a dio mouse model. J. Funct. Food 2015, 14, 623-633. [CrossRef]

70. Dragano, N.R.; Marques, A.; Cintra, D.E.; Solon, C.; Morari, J.; Leite-Legatti, A.V.; Velloso, L.A.; Marostica-Junior, M.R. Freeze-dried jaboticaba peel powder improves insulin sensitivity in high-fat-fed mice. Br. J. Nutr. 2013, 110, 447-455. [CrossRef] [PubMed]

71. Qin, B.; Anderson, R.A. An extract of chokeberry attenuates weight gain and modulates insulin, adipogenic and inflammatory signalling pathways in epididymal adipose tissue of rats fed a fructose-rich diet. Br. J. Nutr. 2012, 108, 581-587. [CrossRef] [PubMed]

72. Seymour, E.M.; Lewis, S.K.; Urcuyo-Llanes, D.E.; Tanone, I.I.; Kirakosyan, A.; Kaufman, P.B.; Bolling, S.F. Regular tart cherry intake alters abdominal adiposity, adipose gene transcription, and inflammation in obesity-prone rats fed a high fat diet. J. Med. Food 2009, 12, 935-942. [CrossRef] [PubMed]

73. Lee, M.; Sorn, S.R.; Park, Y.; Park, H.K. Anthocyanin rich-black soybean testa improved visceral fat and plasma lipid profiles in overweight/obese korean adults: A randomized controlled trial. J. Med. Food 2016, 19, 995-1003. [CrossRef] [PubMed] 
74. Azzini, E.; Venneria, E.; Ciarapica, D.; Foddai, M.S.; Intorre, F.; Zaccaria, M.; Maiani, F.; Palomba, L.; Barnaba, L.; Tubili, C.; et al. Effect of red orange juice consumption on body composition and nutritional status in overweight/obese female: A pilot study. Oxid. Med. Cell. Longev. 2017, 2017. [CrossRef] [PubMed]

75. Silveira, J.Q.; Dourado, G.K.; Cesar, T.B. Red-fleshed sweet orange juice improves the risk factors for metabolic syndrome. Int. J. Food Sci. Nutr. 2015, 66, 830-836. [CrossRef] [PubMed]

(C) 2017 by the authors. Licensee MDPI, Basel, Switzerland. This article is an open access article distributed under the terms and conditions of the Creative Commons Attribution (CC BY) license (http:/ / creativecommons.org/licenses/by/4.0/). 